\title{
Local Area Forcing of Urban-to Regional-Scale Atmospheric Dispersion: Exchanging Fluxes in A Multiscale Enivronment
}

\author{
W.P. Dannevik, S.T. Chan, M.J. Leach, A.A. Mirin
}

February 13, 2003

U.S. Department of Enargy

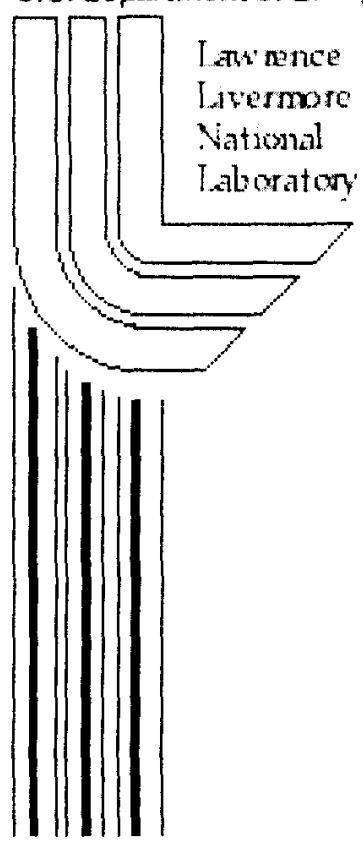




\section{DISCLAIMER}

This document was prepared as an account of work sponsored by an agency of the United States Government. Neither the United States Government nor the University of California nor any of their employees, makes any warranty, express or implied, or assumes any legal liability or responsibility for the accuracy, completeness, or usefulness of any information, apparatus, product, or process disclosed, or represents that its use would not infringe privately owned rights. Reference herein to any specific commercial product, process, or service by trade name, trademark, manufacturer, or otherwise, does not necessarily constitute or imply its endorsement, recommendation, or favoring by the United States Government or the University of California. The views and opinions of authors expressed herein do not necessarily state or reflect those of the United States Government or the University of California, and shall not be used for advertising or product endorsement purposes.

This work was performed under the auspices of the U. S. Department of Energy by the University of California, Lawrence Livermore National Laboratory under Contract No. W-7405-Eng-48.

This report has been reproduced directly from the best available copy.

Available electronically at http://www.doc.gov/bridge

Available for a processing fee to U.S. Department of Energy

And its contractors in paper from

U.S. Department of Energy

Office of Scientific and Technical Information

P.O. Box 62

Oak Ridge, TN 37831-0062

Telephone: (865) 576-8401

Facsimile: (865) 576-5728

E-mail: reports@adonis.osti.gov

Available for the sale to the public from

U.S. Department of Commerce

National Technical Information Service

5285 Port Royal Road

Springfield, VA 22161

Telephone: (800) 553-6847

Facsimile: (703) 605-6900

E-mail: orders@ntis.fedworld.gov

Online ordering: http://www.ntis.gov/ordering.htm

OR

Lawrence Livermore National Laboratory

Technical Information Department's Digital Library

http://www.llnl.gov/tid/Library.html 


\section{Local Area Forcing of Urban- to Regional-Scale Atmospheric Dispersion: Exchanging Fluxes in A Multiscale Environment}

William P. Dannevik, Stevens T. Chan, Martin J. Leach and Arthur A. Mirin

\section{Introduction}

Urban areas are likely locations for release of toxic material into the atmosphere, whether by accident or terrorist act. Both the Department of Energy, through the Chemical and Biological National Security Program, and the Department of Defense, through the Defense Threat Reduction Agency, are supporting simulation and experimental efforts to develop urban modeling capabilities. These developed tools would be used in response to the release of toxic material in populated urban centers.

We are developing a capability to predict the detailed flow and dispersion in and around population centers, to address issues of response to release of toxic agents into the atmosphere. Due to the complexity of these problems and their great demand on computing power, the scientific community has not had the ability to address the urban problem previously. LLNL's unique combination of modeling capability and access to terascale computing resources allows us to address such problems. However, in regions with such small scale features and with heterogeneous building configurations and complex terrain, classical approaches with simplifying assumptions are no longer valid. Turbulence closure approximations that are employed in models with $5 \mathrm{~km}$ resolution are inappropriate when the resolution is 3 orders of magnitude finer. Also, closure assumptions based on vertical transfer are likely not appropriate when the nearest surface is a building face rather than the ground.

The goal of the work described here is to advance the theoretical and empirical basis for transport and dispersion at urban and local scales. This would allow us to extend NARAC's capability for realistic dispersion prediction for smaller scales such as in urban areas. Our objectives within this goal and as part of the mid-year call for proposals are to:

(1) test sub-grid scale (SGS) turbulence approximations in the high-resolution buildingscale model FEM3MP, and determine sensitivity of dispersion predictions to these approximations; and

(2) design procedures for information transfer from a regional scale model COAMPS to the FEM3MP model for prediction purposes, including parallel implementation.

Circulation in the atmosphere is characterized by high Reynolds number, implying that many scales of motion exist; thus direct numerical simulation is not feasible and a Large Eddy Simulation (LES) approach is necessary. In the LES approach, the largest eddies are directly simulated but sub-grid scale motions are modeled. The size of the resolved 
eddies changes as the resolution of a host model changes, but often the SGS model remains unchanged. The resolved flow features and the turbulence intensity depend on that host model resolution. Empirical Monin-Obukhov similarity profiles for neutral conditions provide a good test for the sensitivity to host model resolution.

We investigated resolution issues in the sub-grid scale modeling of turbulence and in the coupling and nesting of models from larger- to smaller-scale domains. These different but related issues are critical in accurately simulating circulation at small scales such as around buildings, street canyons and urban areas. Two numerical models were used in this study: a finite element model, FEM3MP, using a Smagorinsky eddy viscosity formulation for the SGS, and a mesoscale numerical weather prediction model, COAMPS. The coupling of COAMPS to FEM3MP is a one-way nesting, i.e. information passes only from COAMPS to FEM3MP. Time dependent profiles of velocity and turbulence kinetic energy from COAMPS are used to generate, and interpolate if necessary, to provide initial conditions and boundary conditions for FEM3MP.

During the course of this project, we (1) developed protocols and procedures for coupling COAMPS to FEM3MP, (2) conducted mesh refinement studies, (3) tested the COAMPSFEM3MP coupling for fidelity of the information transfer, and (4) compared the FEM3MP LES simulations with data from the Prairie Grass Field Experiment. Comparisons to that dataset included mean horizontal wind profiles, turbulence intensity, and concentration of released tracer as it disperses downwind from the source, as presented in the next section.

\section{Results}

One objective of the present study was to investigate the performance of the sub-grid scale (SGS) turbulence approximations utilized in the building-scale model FEM3MP. To achieve this objective, the LES approach with Smagorinsky SGS turbulence modeling was investigated by performing a series of numerical experiments with varying grid resolutions to simulate a boundary layer flow of the Prairie Grass field experiments (Barad, 1958). The simulated field experiment, No. 20, was conducted under near neutral atmospheric conditions, with a mean velocity of $8.6 \mathrm{~m} / \mathrm{s}$ at $2-\mathrm{m}$ elevation and a friction velocity of $0.6 \mathrm{~m} / \mathrm{s}$. The roughness length at the site was estimated to be $0.6 \mathrm{~cm}$.

Numerical experiments were first conducted to determine the size of computational domain required for the flow being simulated. To this end, a domain of $1600 \mathrm{~m} \mathrm{x} 800 \mathrm{~m} \mathrm{x}$ $400 \mathrm{~m}$ in the longitudinal, lateral, and vertical directions, respectively, and a smaller domain of $1000 \mathrm{~m} \times 400 \mathrm{~m} \times 200 \mathrm{~m}$, both having the same grid resolutions, were employed in this investigation. The overall results, including velocity, turbulent kinetic energy (TKE) profiles and other turbulence statistics, from the two simulations were observed to agree quite well, suggesting that the smaller domain is adequate for the following grid resolution study.

With the selected domain size, simulations with three grids representing coarse, medium, and fine resolution, respectively, were performed. The medium grid has resolution of 20 
$\mathrm{m}$ on the horizontal plane and $5 \mathrm{~m}$ in the vertical direction. The coarse grid has twice the above grid spacing, and the fine grid has one-half of the above grid spacing. In all simulations, periodic boundary conditions were used in both horizontal directions, together with no-slip boundary condition on the ground surface and no penetration on the top boundary. A constant pressure gradient of $1.0 \mathrm{~Pa} / \mathrm{km}$, inferred from contour maps of the surface pressure data, was used to drive the flow. In addition, the wall damping function devised by Piomelli, et al. (1987) was used to model the viscous sub-layer near the ground surface. All three cases of simulation appear to perform satisfactorily except that results are somewhat sensitive to the vertical extent over which the wall damping function is applied. After some numerical experimentation, the lengths of $10 \mathrm{~m}, 15 \mathrm{~m}$, and $20 \mathrm{~m}$ (for the fine, medium, and coarse grids), respectively, were selected for applying the wall damping function. Overall results, including velocity and TKE fields, and certain turbulence statistics from simulations using the fine and medium grids, are very similar and highly consistent with the observed data; results from the coarse grid are less accurate but are still consistent with field measurements. The simulated results suggest that the medium resolution grid is adequate for the boundary layer flow under consideration.

In Figure 1, vertical profiles of velocity, TKE, and turbulent shear stress obtained from the medium resolution grid for the last hour of a16-hour simulation are 
(a) Instantaneous u-velocity profiles

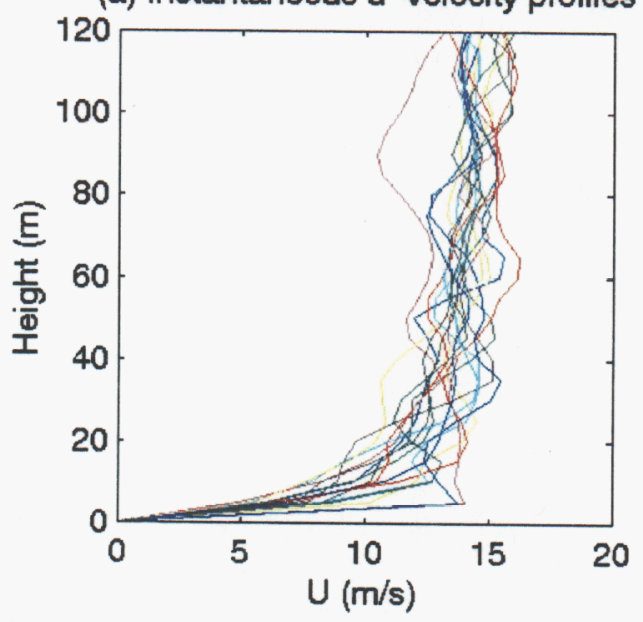

(c) Mean TKE profile

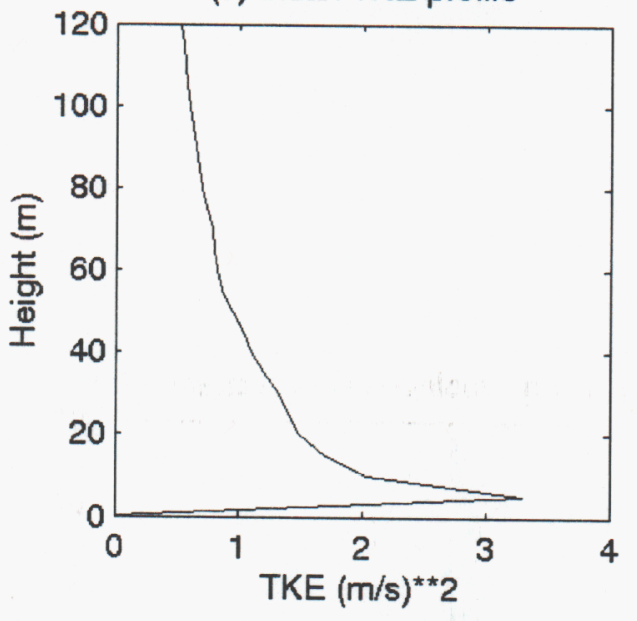

(b) Predicted mean u-velocity vs log profile

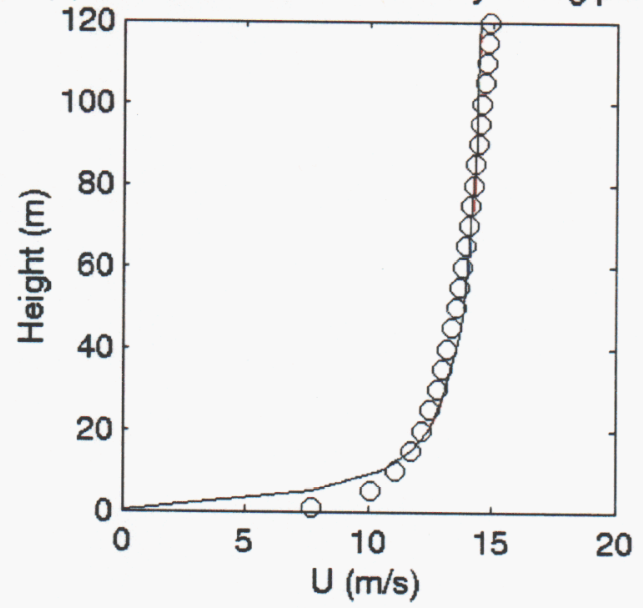

(d) Mean shear stress profile

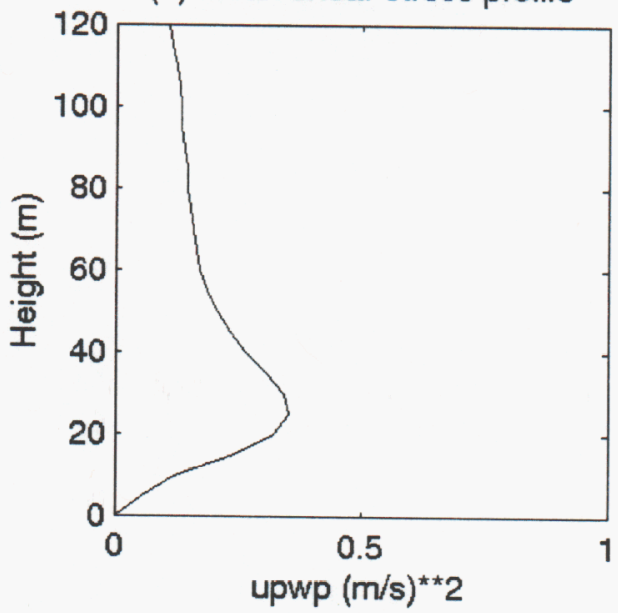

Figure 1. Vertical profiles of (a) instantaneous horizontal wind velocity, (b) mean horizontal wind velocity (with observation data plotted as circles, (c) mean Turbulence Kinetic Energy, and (d) turbulent shear stress for FEM3MP simulations with pressure gradient specified to represent large scale conditions. 
presented. In panel (a), the instantaneous u-velocity profiles at a representative location are shown to illustrate the large extent of turbulent velocity fluctuations. In panel (b), the mean velocity profile, which was obtained by time averaging of the instantaneous velocity profiles over the last hour, is compared with a log profile (in circles) constructed from the measured data. The agreement between model prediction and measured data is very good except near the ground surface, where a slight under-prediction by FEM3MP occurs. In the last two panels, the predicted mean TKE and shear stress profiles are displayed, which are typical of a boundary layer flow under neutral atmospheric conditions. As a further measure of the model's performance, turbulence intensities for the three velocity components were also evaluated for the last hour of simulation. The predicted values are $2.51,1.15,0.77$ at $\mathrm{z}=20 \mathrm{~m}$ and $1.77,1.07,1.01$ at $\mathrm{z}=50 \mathrm{~m}$, respectively. These values are in reasonable agreement with the observed values of 2.42 , $1.73,1.25$, of which the first two numbers pertain to the estimates based on all Prairie Grass experiments and the last number is an average value from many observations at various locations characterized by boundary layer flows over similar, uniform terrain (Panofsky and Dutton, 1984, p160).

Another objective of the present study was to develop procedures for information transfer from COAMPS, a mesoscale model, to FEM3MP, a building-scale model. There are several issues related to coupling FEM3MP to COAMPS. The models employ different numerical schemes, the model equation sets are not exactly the same, and the methods for approximating turbulence are inherently different. During the course of this project, we investigated the effects of passing turbulence from COAMPS to FEM3MP. Turbulence in COAMPS is modeled using a Reynolds-Averaged Navier-Stokes (RANS) approximation. In the RANS approach, all turbulence is approximated with a closure scheme where production of turbulence is estimated based on mean quantities for wind shear and buoyancy. This contrasts with an LES scheme, where only sub-grid scale turbulence is parameterized and the larger scale eddies are explicitly resolved. There are several RANS closure schemes that have been formulated. The current version of COAMPS uses a scheme suggested by Mellor and Yamada (1974).

We performed several simulations with COAMPS to test the sensitivity of the turbulence intensity that is generated to the model grid spacing. The initial conditions were defined by specifying a constant pressure gradient force (PGF) equivalent to approximately 1.0 $\mathrm{Pa} / \mathrm{km}$. The PGF and temperature profile were defined using conditions from the Prairie Grass field experiments. Our finest scale simulations were performed using $\Delta x=\Delta y=100$ meters, $\Delta z=10$ meters. The maximum intensity of turbulence generally increases as resolution increases, although there are only minor differences when the simulations are half the finest resolution $(\Delta x=\Delta y=200 \mathrm{~m}, \Delta \mathrm{z}=20 \mathrm{~m})$. Results from the finest scale simulation are shown in Figure 2. The turbulence kinetic energy (TKE) per unit mass is plotted as a function of height above the ground, 300 hours into a simulation as the solution approaches steady state. The maximum value occurs very close to the ground at about 70 to 80 meters. This non-slip lower boundary generates wind shear near the surface, as the winds in the atmosphere are maintained by the specified PGF. The shape of the profile of TKE appears reasonable, but the maximum intensity is too low, relative to that calculated in the FEM3MP runs. This maximum value that is too low 
creates an unresolved issue for coupling COAMPS to FEM3MP by transferring timedependent boundary conditions that include profiles of the mean wind with estimates of turbulence intensity.

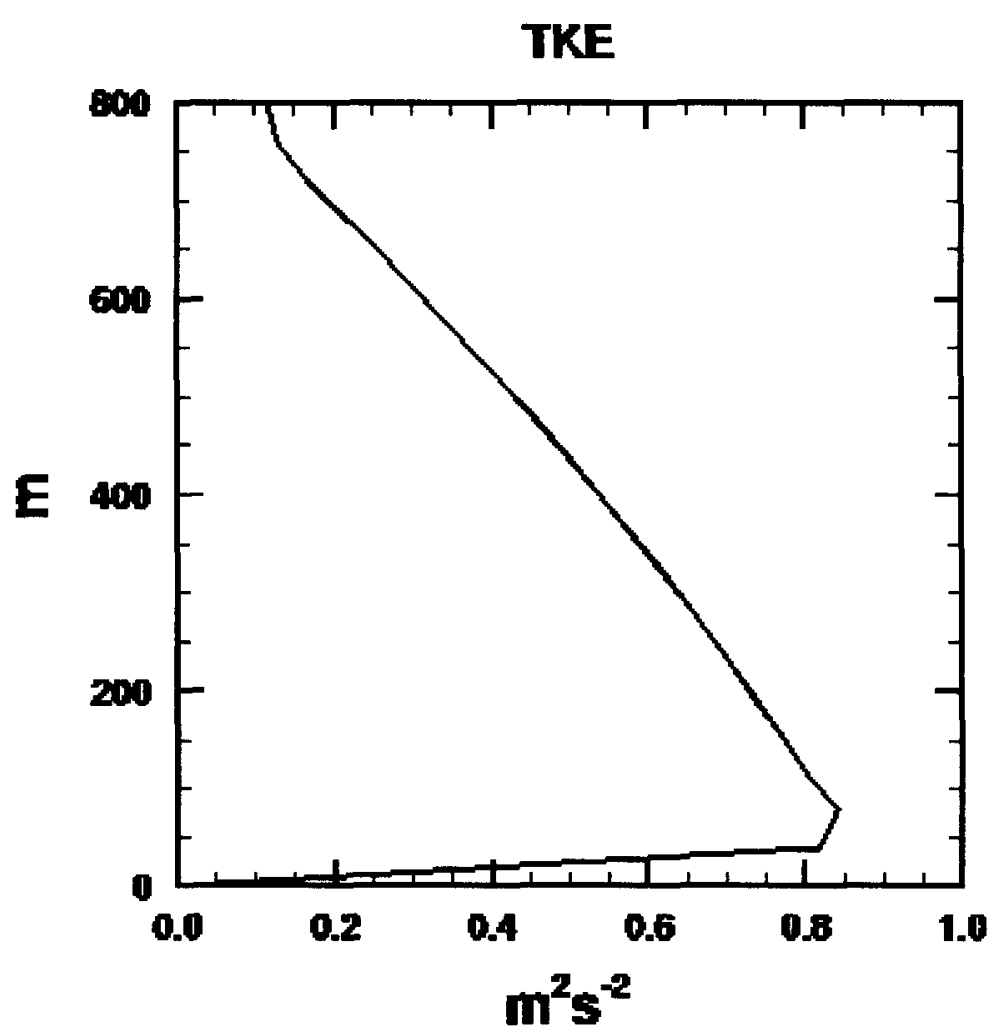

Figure 2. The Turbulence Kinetic Energy profile as a function of height produced in a COAMPS simulation

Time dependent profiles of velocity and TKE from COAMPS were used to generate appropriate initial and boundary conditions for the FEM3MP simulation, using the medium resolution grid in FEM3MP. Specifically, the velocity and TKE profiles (which are generally functions of time) from COAMPS were 'combined', making use of random perturbations, to generate instantaneous velocity components and used as Dirichlet boundary conditions on the inlet plane of the FEM3MP simulation. As a result, a periodic boundary condition is invoked only in the lateral direction. Since the flow is currently being driven by time-dependent velocity on the inlet plane, the constant pressure gradient (representing large scale forcing) used in the earlier simulations is no longer needed. The simulation was performed for 4 hours and it was found that the wall damping function has to be applied to $75 \mathrm{~m}$ above the ground surface in order to generate sufficient level of turbulence in the flow field.

In Figure 3, profiles of instantaneous velocity, mean velocity, TKE, and shear stress are displayed from the simulation where the large-scale forcing is included by passing the 
time-dependent boundary conditions from a COAMPS simulation. Figure 3(b) indicates the predicted mean velocity profile is in fair agreement with the log profile constructed from the measured data. However, the model largely under-predicts turbulence, in particular the shear stress, in view of the results shown in Fig. 1. For turbulence intensity,

(a) Instantaneous u-velocity profiles

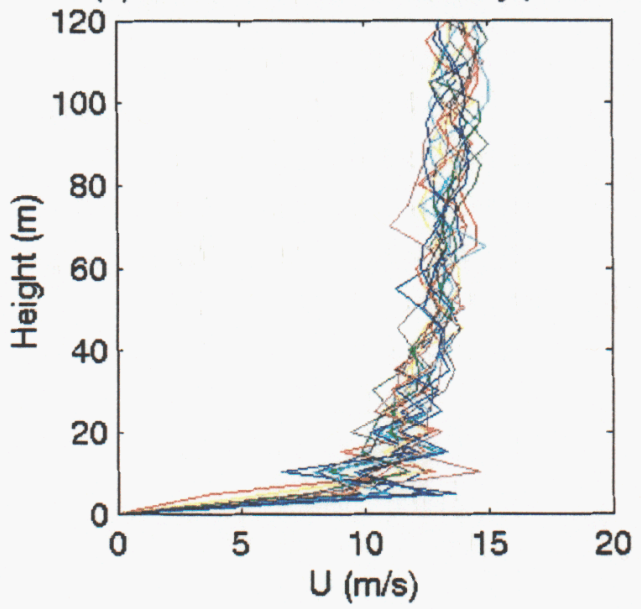

(c) Mean TKE profile

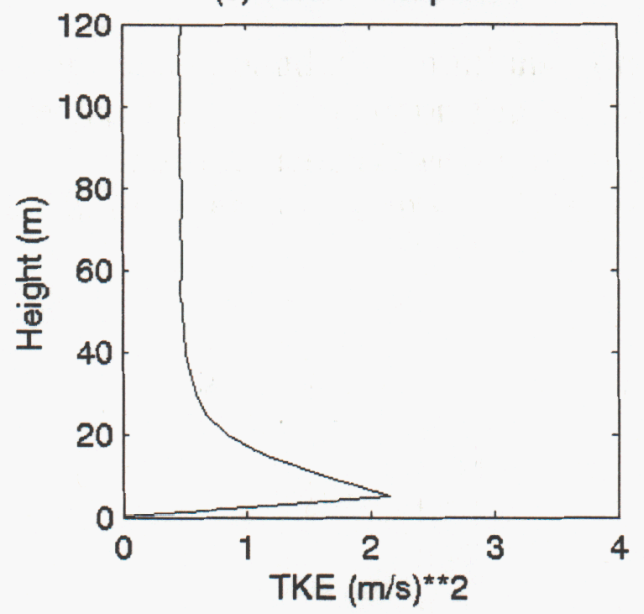

(b) Predicted mean u-velocity vs log profile

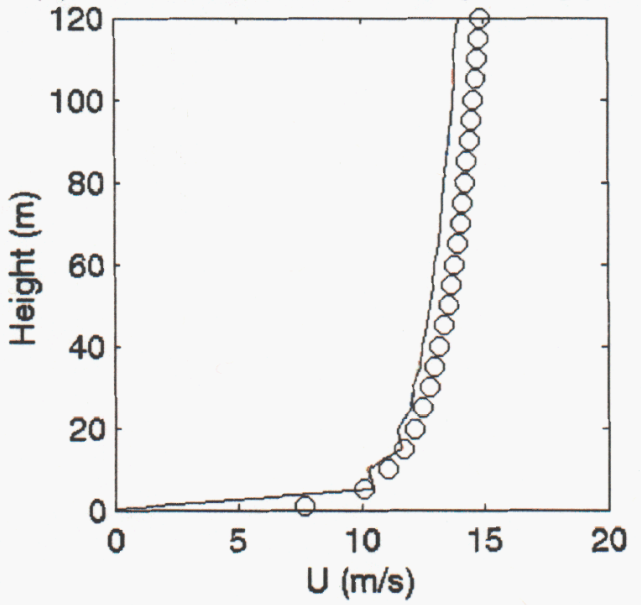

(d) Mean shear stress profile

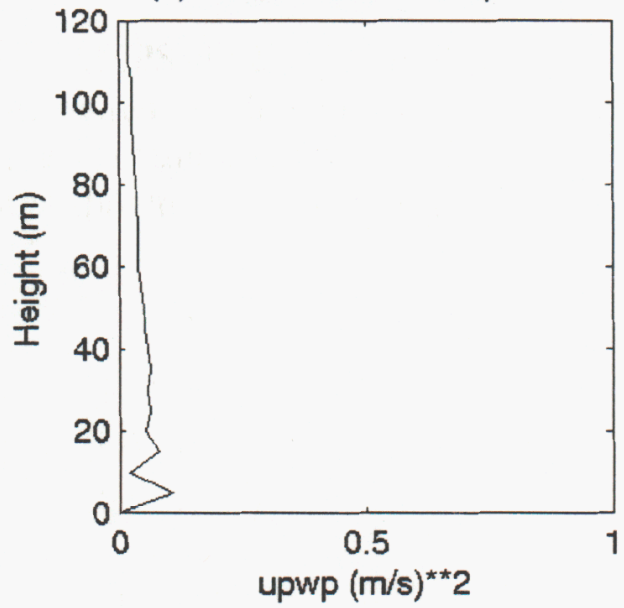

Figure 3. Vertical profiles of (a) instantaneous horizontal wind velocity, (b) mean horizontal wind velocity (with observation data plotted as circles, (c) mean Turbulence Kinetic Energy, and (d) turbulent shear stress for FEM3MP simulations with large scale forcing represented by passing boundary conditions from COAMPS.

the present simulation yields values of $1.75,1.11,0.57$ at $\mathrm{z}=20 \mathrm{~m}$ and $1.25,0.80,0.59$ at $\mathrm{z}=50 \mathrm{~m}$, which are reasonable but are generally inferior to those predicted by the simulation without coupling with COAMPS. The main cause of under-prediction of turbulence in the present simulation is due to the fact that the TKE profile provided by 
COAMPS is much smaller than expected, which in turn results in much smaller fluctuating velocity on the inlet plane in the FEM3MP simulation. COAMPS is a RANS (Reynolds-Averaged Navier-Stokes) model and its TKE field is not completely consistent with that of an LES model. A TKE field generated by an LES SGS turbulence model is more appropriate but remains to be developed in COAMPS.

We are probably the first to have successfully simulated both the flow field and tracer dispersion for experiments such as Prairie Grass, using the LES approach. The predicted instantaneous velocity and tracer concentration (with contour levels in log scale) on two representative planes, are shown in Figure 4, with a comparison of the LES predicted peak concentration with measured data from Prairie Grass Experiment No. 20.and with predictions using a Gaussian model. The agreement between the LES results and the data, especially as compared to the Gaussian model results, is excellent. This example illustrates that, even for a case involving flat terrain, a sophisticated computational fluid dynamics model is needed in order to obtain accurate results in the near field, let alone in the case of an urban area involving variable terrain and many buildings.

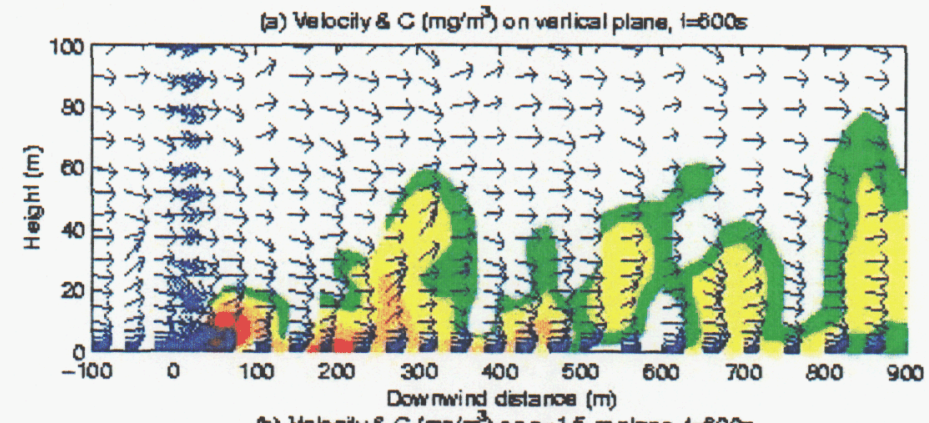

(B) Vabcily \& C (mg: $\left.\mathrm{m}^{3}\right)$ on $z=1.5 \mathrm{~m}$ plane, $1=800 \mathrm{~s}$

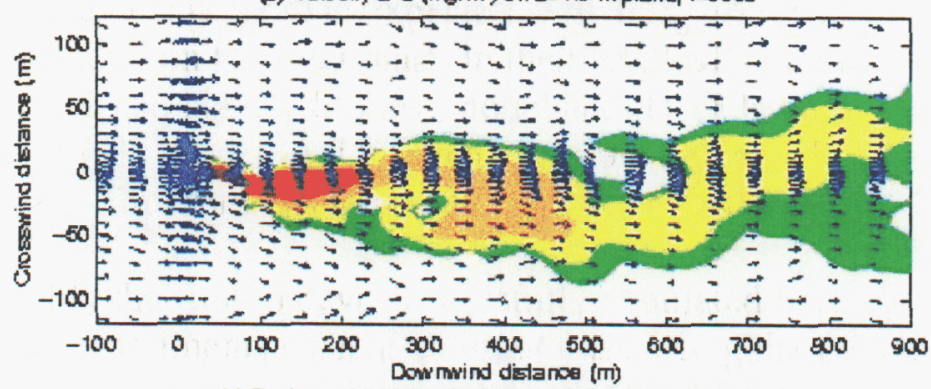

(c) Peak concentration in downwind direction tor $1=0-600 \mathrm{~s}$

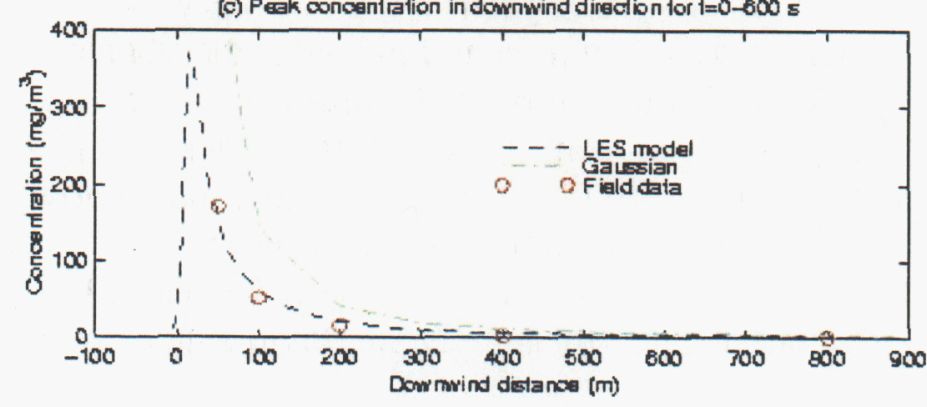

Figure 4. A vertical slice and a plan view of the predicted concentration using the LES model are in the top and middle frame. The vertical slice is along the plume centerline and the plan view is at 1.5 meters above ground. The bottom frame is the maximum predicted concentration from the LES model (dashed, blue lines) and from the Gaussian model (dot-dashed, green). The observed values are plotted as red, open circles. 


\section{Conclusions and Future Work}

FEM3MP, when specifying the large scale forcing by using the observed pressure gradient force at the time of the Prairie Grass experiment, simulates the observed profile of the mean wind reasonably well, with turbulence intensities of the right magnitude. This suggests that the sub-grid scale turbulence model in the LES simulations performs adequately. However, when using the COAMPS simulations for large scale forcing in FEM3MP, the turbulence intensity forecast in FEM3MP appears to be too low. This is most likely due to the inadequate representation of turbulence by the COAMPS RANS model, which is designed for larger-scale (and coarser resolution) simulations. In this sixmonth LDRD project, we investigated this key issue of turbulence transfer when coupling the two models.

The use of Smagorinsky SGS turbulence model in FEM3MP requires a priori specification of the model coefficient and the length scale, which have to be modified via a wall damping function to account for near-wall effects. Although the results are generally satisfactory, they are rather sensitive to the vertical extent over which the wall damping function is applied. To overcome these drawbacks, Germano et al. (1991) developed a dynamic SGS model, of which the model coefficient is determined as the simulation progresses and it is capable of taking into account the near-wall effects without requiring damping of the length scale near the wall.

Our next steps would include implementing and testing a more advanced SGS turbulence model in FEM3MP, facilitating the coupling between COAMPS and FEM3MP by incorporating an LES option in COAMPS, and further evaluating the modeling system using datasets from experiments in more complex environments.

\section{References}

Barad, M.L.,ed, 1958: 'Project Prairie Grass, a Field Program in Diffusion' (three Volumes), Geophysical Research Papers, 73, AFCRC-TR-58-235.

Germano, M., U. Piomelli, P. Moin, and W. Cabot (1991): 'A Dynamic Subgrid-Scale Eddy Viscosity Model', Phys. Fluids A 3 (7), 1760-1765.

Mellor, G. and T. Yamada, 1974: A hierarchy of turbulence closure models for planetary boundary layers. J. Atmos. Sci., 31,1791-1806.

Panofsky, H.A. and J.A. Dutton (1984): Atmospheric Turbulence, John Wiley \& Sons.

Piomelli, U., J. Ferziger, and P. Moin (1987): 'Models for Large Eddy Simulations of Turbulent Channel Flows Including Transpiration', Report TF-32, Dept. of Mech. Engr., Stanford University. 